\title{
Gaining the upper hand on COVID-19 misinformation
}

Alex Cen ${ }^{1}$ and Lara Parlatan ${ }^{2}$

\author{
${ }^{1}$ Faculty of Health Sciences, McMaster University, Ontario, Canada \\ ${ }^{2}$ Department of Pathology and Laboratory Medicine, Schulich School of Medicine and Dentistry, University of Western \\ Ontario, Ontario, Canada
}

KEYWORDS: infodemic, COVID-19, misinformation, social media, infodemiology

\section{INTRODUCTION}

As the Coronavirus Disease 2019 (COVID-19) pandemic evolved, information about the virus also accumulated. However, accompanied by the quick emergence of factual information was an even greater abundance of false information. For example, by March 2020, videos containing non-factual information on COVID-19 accounted for over one-quarter of the most viewed videos on YouTube - greatly exceeding the popularity of factual videos released by governments and health professionals [1]. The World Health Organization declared this massive flux of misinformation surrounding COVID-19 an "infodemic", where it is hard to distinguish between factual and non-factual information [2].

There is a need to curb the spread of misinformation; fake claims about treatments can endanger lives, and misinformation in the form of conspiracy theories can lead people to disregard government health guidelines [3]. Moreover, narratives promoted by misinformation often create mistrust in institutions and health officials, which poses a hurdle to public health and efforts to contain the spread of COVID-19 by decreasing adherence to health measures [4]. Some strategies to combat online misinformation have been employed throughout this pandemic, such as fact-check labels next to misinformed posts on social media platforms [5]. These tactics aim to warn people of potentially misleading information, but their effectiveness is debated [6-9]. As such, this viewpoint proposes new strategies and changes that health officials could implement to combat the growing misinformation surrounding COVID-19.

Ourpaper borrows definitions from Wardle and Derakhshan (2017), who define misinformation as false information that is spread without intent to cause harm. Additionally, our paper will only focus on micro-level transmission, which is when misinformation is transferred between individuals [10]. As the landscape of the COVID-19 infodemic is constantly changing, our viewpoint draws most of its conclusions based on past infodemics, such as those surrounding previous pandemics, or misinformation surrounding vaccines.

\section{THE SPREAD OF MISINFORMATION}

Misinformation spreads through a variety of platforms, including social media sites, blog posts, and mainstream media [11]. It propagates fastest through polarized news sources or personal blogs, which act as echo chambers that exploit people's selective exposure [12]. Additionally, false information often spreads faster and farther than factual information [4]. In a study conducted by Vosoughi et al. [13], authors found false news stories on Twitter travelled significantly faster and reached a far wider audience than accurate news stories. Similar findings were presented in a meta-analysis by Wang et al. [4] in which the authors analyzed the spread of information on various social media platforms about the link between the MMR vaccine and autism. They determined that although there were more correct than incorrect information, incorrect information still gained more views [4]. In contrast to dynamics seen with information surrounding vaccines, $\mathrm{Li}$ et al. [1] found misinformation surrounding COVID-19 outnumbered factual information on YouTube. As expected, videos containing factual information only made up $10 \%$ of views from all videos they analyzed [1], which suggests that health officials should work harder to disseminate more factual COVID-19 information on the internet. These studies demonstrate that misinformation has an advantage over evidence-based information, which allows misinformation to spread quicker even when a greater proportion of correct information is present.

\section{WHY IS MISINFORMATION MORE INFECTIOUS?}

A key advantage that misinformation has over factual 
information is its ability to appeal to emotion and a person's beliefs $[4,16]$. Sell et al. [14] examined tweets made on Twitter during the 2014 Ebola crisis and found that posts containing misinformation often also contained strong political views and fear-inducing elements. Their findings are consistent with previous studies which show that misinformation is often tied to political views, conspiracy theories, and preexisting beliefs held by certain groups of people [4, 15]. Notably, the same piece of misinformation can easily appeal to a variety of different groups, and people who believe in one conspiracy theory often believe in multiple other conspiracies addressing different topics, such as those of climate change, governments, and vaccination [3]. This is likely due to the greater malleability of misinformation over factual information, whereby the same narrative can be easily adapted to target different groups. Misinformation can also incite fear, and a wide range of studies have shown that feeling threatened or anxious reduces effective decision making and analytic thinking [15-17]. For example, Wu discussed how decision making was skewed by anxiety induced by the time it takes to see the outcome of a gamble [18], and Swami et al. [19] demonstrated that analytic thinking reduces belief in conspiracy theories. Together, these studies suggest that reducing public anxiety or promoting analytic thinking may be an effective way to combat misinformation.

\section{WINNING THE UPHILL BATTLE}

\section{Disseminate information via trusted individuals}

Scientists providing knowledge can turn the tide in the battle against misinformation by utilizing techniques that give false information an advantage in the first place. Similar to how misinformation spreads through an appeal to existing beliefs, factual information can gain the upper hand by being disseminated through trusted members of a community [20]. For example, public health officials can encourage established COVID-19 guidelines and recommendations to be distributed by local religious leaders to increase compliance [20].

\section{Focus on the error}

Additionally, efforts to debunk existing beliefs in misinformation should focus on the error in the information itself and not the personal views associated with the belief. Focusing on the error rather than personal views requires scientists to cooperate with the media, as coverage that emphasizes politics rather than the error while debunking a statement made by a political leader polarizes people and reinforces their beliefs [21]. When Paynter et al. [22] compared participant support for unproven autism treatments after different styles of debunking, they found that appeals to credibility and self-affirmation were more effective in convincing people than fact-vs-myth debunking. The results of the study are illustrated in Figure 1, with the control group exposed to fact-vs-myth debunking while the proposed debunking was implemented in the experimental group [22].

\section{Messaging through individual scientists}

Furthermore, factual information and debunking messages should be promoted by individual scientists, not just institutions. When individual scientists promote information, a small group dynamic is created which permits interactions between scientists and the public to persist longer [23]. This small group dynamic may be better to communicate with people who lack trust in institutions [20].

Implementing these recommendations will require coordination between academics, public health officials, government, and media. While such an effort may seem intensive, it is important to note that similar initiatives in the past have been beneficial. For example, Ireland experienced a drastic decline in its Human Papillomavirus (HPV) vaccination rates in $2015-2017$ from $87 \%$ to $50 \%$, due to lobby groups advertising strong personal narratives and misinformation through mainstream media [24]. To combat the lobby groups' work, officials launched the HPV Vaccination Alliance in 2017, where various organizations appealing to different beliefs banded together to raise awareness and promote HPV vaccines. As a result, the vaccination rate increased to $62 \%$ in the following year [24].

Furthermore, initiatives led by public health may not need to convince everyone. Research has shown that a sufficiently sized minority with a differing view can overturn the consensus in a group with an established belief and change it in their favour [25]. As misinformation often persists in specific groups, efforts may only need to target a small proportion of a group regarding the falseness of certain information through debunking before the majority of the group buys in. Interventions must also be designed and adapted as what may work for one group may not work for another. One way 


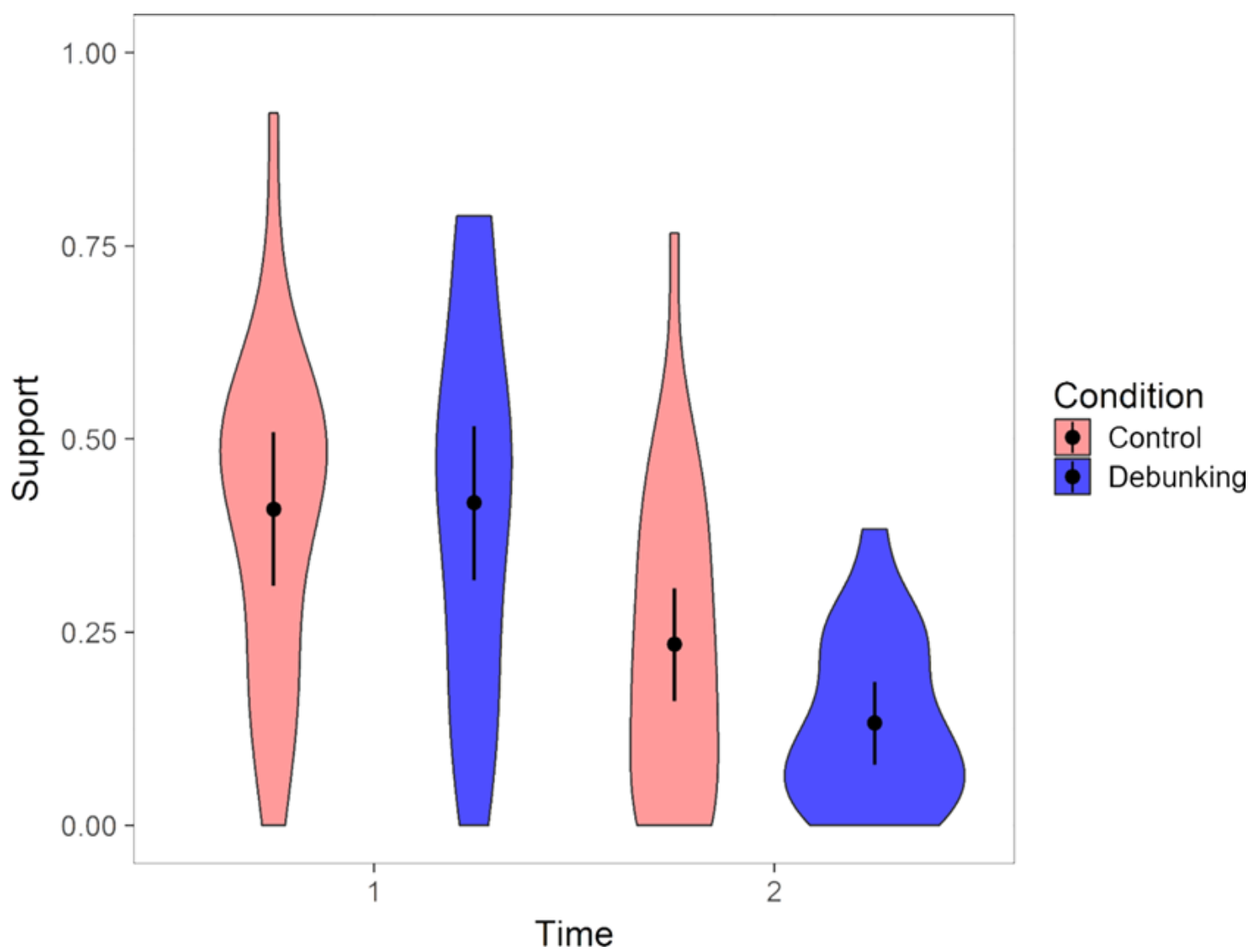

Figure 1. Violin plot showing support for unproven autism treatments before (time 1) and after (time 2) debunking, with time intervals on the $\mathrm{x}$-axis and median support for unproven treatments on the $y$-axis. Error bars show the $95 \%$ confidence intervals. Original figure from Paynter et al. [22] in PLOS One and reproduced under CC BY 4.0 license.

for public health to find out what could work for specific groups is to analyze social media trends. For example, during the 2009 H1N1 outbreak, Chew and Eysenbach (2010) used content analysis of tweets on Twitter to measure public attitudes and frustration towards the H1N1 pandemic. The authors argued that such infodemological studies may help health authorities identify and respond to concerns from the public [26].

Strategies which have been successful for pandemics like HIV/AIDS and H1N1 may work even better for COVID-19, due to how much COVID-19 has affected daily life globally through lockdowns and public policy [2]. People may be more likely to discuss their perspectives surrounding COVID-19 with those they trust, which reaffirms the importance that the public receives correct information from trusted community leaders. Moreover, as COVID-19 has been much more politicized than past pandemics [9], it is even more important that debunking efforts focus on the belief itself. The role of individual scientists in countering misinformation is even more salient today due to greater public social media presence and activity [27]. Health messaging may benefit from adapting similar strategies from past epidemics.

\section{CONCLUSION}

The COVID-19 pandemic presents a unique situation where the public must distinguish truth from hoax in the face of copious amounts of information. To combat the spread of misinformation, new approaches are required. Alongside existing fact-checking labels beside social media posts, governments could also work with local community leaders to disseminate health guidelines for increased compliance. Messaging that debunks misinformation can also be promoted by scientists through online platforms to appeal to individuals who do not trust larger institutions. Furthermore, initiatives to debunk existing misinformation could avoid polarization by focusing on the error in the misinformation, rather than the beliefs associated with it. Media outlets can also play a role by cooperating with health officials, not only to 
ensure that common misconceptions are debunked, but that accurate information receives appropriate news coverage. These initiatives should emphasize increasing public trust in health officials and governments, as misinformation commonly appeals to those who do not have confidence in governing bodies. The impacts of the COVID-19 infodemic have shown governments and health officials around the world that the spread of misinformation should be addressed properly through new approaches. The effectiveness with which governments manage this infodemic will greatly influence the course of current and future disease outbreaks.

\section{ACKNOWLEDGEMENTS}

A special thank you to Lara Parlatan for her guidance and dedication. This paper was written as part of the 2020 STEMpowerment Viewpoint Competition.

\section{REFERENCES}

1. Li HO-Y, Bailey A, Huynh D, Chan J. YouTube as a source of information on COVID-19: A pandemic of misinformation? BMJ Global Health. 2020;5(5).

2. World Health Organization. Novel Coronavirus (2019nCoV) Situation Report-13. Geneva; February 2, 2020. Available from https://www.who.int/docs/defaultsource/coronaviruse/situation-reports/20200202-sitrep13-ncov-v3.pdf

3. Freeman D, Waite F, Rosebrock L, Petit A, Causier C, East A, et al. Coronavirus conspiracy beliefs, mistrust, and compliance with government guidelines in England. Psychological Medicine. 2020;1-13.

4. Wang Y, Mckee M, Torbica A, Stuckler D. Systematic literature review on the spread of health-related misinformation on social media. Social Science \& Medicine. 2019;240:112552.

5. Pennycook, G., Bear, A., Collins, E. T., \& Rand, D. G. (2020). The implied truth effect: Attaching warnings to a subset of fake news headlines increases perceived accuracy of headlines without warnings. Management Science. doi:10.1287/mnsc.2019.3478

6. Chan M-PS, Jones CR, Jamieson KH, Albarracín D. Debunking: A meta-analysis of the psychological efficacy of messages countering misinformation. Psychological Science. 2017;28(11):1531-46.

7. Banas JA, Miller G. Inducing resistance to conspiracy theory propaganda: Testing inoculation and metainoculation strategies. Human Communication Research. 2013;39(2):184-207.

8. Zarocostas J. How to fight an infodemic. The Lancet. 2020;395(10225):676.

9. Orso D, Federici N, Copetti R, Vetrugno L, Bove T.
Infodemic and the spread of fake news in the COVID19-era. European Journal of Emergency Medicine [Internet]. 2020. Available from https://doi.org/10.1097/ MEJ.0000000000000713

10. Wardle C, Derakhshan H. [Internet]. Information disorder: Toward an interdisciplinary framework for research and policy making. Council of Europe; Sep 27, 2017. Available from: https://edoc.coe.int/ en/media/7495-information-disorder-toward-aninterdisciplinary-framework-for-research-and-policymaking.html

11. Lewandowsky S, Ecker UKH, Seifert CM, Schwarz N Cook J. Misinformation and its correction. Psychological Science in the Public Interest. 2012;13(3):106-31.

12. Stroud NJ. Polarization and partisan selective exposure. Journal of Communication. 2010;60(3):556-76.

13. Vosoughi S, Roy D, Aral S. The spread of true and false news online. Science. 2018;359(6380):1146-51.

14. Sell TK, Hosangadi D, Trotochaud M. Misinformation and the US Ebola communication crisis: analyzing the veracity and content of social media messages related to a fear-inducing infectious disease outbreak. BMC Public Health. 2020;20(1).

15. Jamieson $\mathrm{KH}$, Albarracín $\mathrm{D}$. The relation between media consumption and misinformation at the outset of the SARS-CoV-2 pandemic in the US. Harvard Kennedy School Misinformation Review [Internet]. 2020; Available from: https://doi.org/10.37016/mr-2020-012

16. Slovic $P$, Finucane $M L$, Peters $E$, Macgregor DG. Risk as analysis and risk as feelings: Some thoughts about affect, reason, risk, and rationality. Risk Analysis. 2004;24(2):311-22.

17. Rottenstreich Y, Hsee CK. Money, kisses, and electric shocks: On the affective psychology of risk. Psychological Science. 2001;12(3):185-90.

18. Wu G. Theory and Decision. 1999;46(2):159-99.

19. Swami V, Voracek M, Stieger S, Tran US, Furnham A Analytic thinking reduces belief in conspiracy theories. Cognition. 2014;133(3):572-85.

20. Bavel JJV, Baicker K, Boggio PS, Capraro V, Cichocka A, Cikara $M$, et al. Using social and behavioural science to support COVID-19 pandemic response. Nature Human Behaviour. 2020;4(5):460-71.

21. Cook J, Lewandowsky S, Ecker UKH. Neutralizing misinformation through inoculation: Exposing misleading argumentation techniques reduces their influence. Plos One. 2017;12(5).

22. Paynter J, Luskin-Saxby S, Keen D, Fordyce K, Frost G, Imms C, et al. Evaluation of a template for countering misinformation-Real-world Autism treatment myth debunking. Plos One. 2019;14(1).

23. Palla G, Barabási A-L, Vicsek T. Quantifying social group evolution. Nature. 2007;446(7136):664-7.

24. Corcoran B, Clarke A, Barrett T. Rapid response 
to HPV vaccination crisis in Ireland. The Lancet. 2018;391(10135):2103.

25. Centola D, Becker J, Brackbill D, Baronchelli A. Experimental evidence for tipping points in social convention. Science. 2018;360(6393):1116-9.

26. Chew C, Eysenbach G. Pandemics in the age of twitter: Content analysis of tweets during the $2009 \mathrm{H} 1 \mathrm{~N} 1$ outbreak. PLoS ONE. 2010;5(11).

27. Johnson NF, Velásquez N, Restrepo NJ, Leahy R, Gabriel N, El Oud S, et al. The online competition between pro- and anti-vaccination views. Nature. 2020 Jun;582(7811):230-3. 